\title{
Acquisition and eradication of $P$. aeruginosa in young children with cystic fibrosis
}

\author{
T.A. Douglas*,\#, S. Brennan\#, S. Gard" ${ }^{\#}$ L. Berry", C. Gangell ${ }^{\#}$, S.M. Stick*, \\ B.S. Clements* and P.D. Sly*\#
}

ABSTRACT: When do infants and young children with cystic fibrosis acquire infection with Pseudomonas aeruginosa? Can this be eradicated when first detected?

Children $<6$ yrs of age participated in an annual bronchoalveolar lavage (BAL)-based microbiological surveillance programme in Perth, Australia. When $P$. aeruginosa was detected, an eradication programme using combination treatment with i.v., oral and nebulised antibiotics was undertaken. Repeat BAL was performed 3 months following treatment, to assess eradication success.

$P$. aeruginosa was detected in $33(28.4 \%)$ children; median (range) age at detection was 30.5 (3.3-71.4) months. $P$. aeruginosa was mucoid at detection in six (18.2\%) out of 33 patients and associated with respiratory symptoms in 16 (48.5\%) out of 33 children. In total, 26 children underwent eradication therapy, with P. aeruginosa eradicated in 20 (77\%) out of 26 following one eradication cycle and in three (total $88 \%$ ) additional children following a second cycle. Eradication was associated with a significant decrease in neutrophil elastase and interleukin-1 $\beta$ in BAL fluid 12 months post eradication.

Eradication of Pseudomonas aeruginosa infection is achievable in young children with cystic fibrosis for up to $\mathbf{5}$ yrs using combination i.v., oral and nebulised antibiotic therapy and is associated with reduced pulmonary inflammation 12 months post eradication.

KEYWORDS: Bronchoalveolar lavage, children, cystic fibrosis, eradication, microbiological surveillance, Pseudomonas aeruginosa

ung disease in cystic fibrosis (CF) begins early in life [1-5], with a Pseudomonas aeruginosa infection associated with a worse prognosis [6-11]. Some clinics go to great lengths to eradicate $P$. aeruginosa when first detected [10-17]. National CF registries report that the prevalence of a $P$. aeruginosa infection in preschool children is 9-30\% [18-20]. However, these data are based mainly on upper airway swabs or collections during respiratory exacerbations. Population-based data, describing when children first develop pulmonary infections and what organisms are involved, are lacking. Young children can rarely expectorate sputum and the poor positive predictive values of oropharyngeal swabs, cough swabs or cough plates for pulmonary infection compound this deficiency [21, 22]. Previous studies have reported detection of the $P$. aeruginosa infection in the lower airways using bronchoalveolar lavage (BAL), with between 0 and $45 \%$ of children studied being infected $[2,11$, 12]. However, these reports have not included all children from the respective populations; thus, the population prevalence of $P$. aeruginosa infection and the age of acquisition are uncertain.
In 1999, the Princess Margaret Hospital CF clinic in Perth, Australia, introduced a bronchoscopyand BAL-based microbiological surveillance programme for all children attending the clinic up to 6 yrs of age or until they could expectorate sputum. The aim of the present study was to determine the population prevalence and age of acquisition of pulmonary infection with $P$. aeruginosa. In addition, the current authors report the effect of combined parenteral/oral and nebulised anti-Pseudomonas therapy on $P$. aeruginosa eradication.

\section{METHODS}

Children born prior to the introduction of newborn screening (NBS) in 2000 and those detected by NBS had the diagnosis of CF confirmed by a sweat test, CF genotyping and clinical features. Each child underwent BAL at diagnosis (within the first 6-12 weeks after birth) and annually until the age of 6 yrs or until the child was able to expectorate sputum. Additional BALs were performed following $P$. aeruginosa eradication therapy. Parents were specifically asked about the presence of respiratory symptoms (cough,
AFFILIATIONS

*Dept of Respiratory Medicine Princess Margaret Hospital, and "Division of Clinical Sciences, Telethon Institute for Child Health Research, Centre for Child Health Research, University of Western Australia, Perth, Australia.

CORRESPONDENCE P.D. Sly Telethon Institute for Child Health Research

Centre for Child Health Research University of Western Australia Perth Western Australia 6008

Fax: 61894897706

E-mail: peters@ichr.uwa.edu.au

Received:

March 192008

Accepted after revision:

October 122008

\section{SUPPORT STATEMENT}

Grants for the present study were received from the National Health and Medical Research Council (grants 211912 and 458513; Canberra, Australia) and the US Cystic Fibrosis Association (grant SLYO4AO; Bethesda, MD, USA).

STATEMENT OF INTEREST

None declared. 
wheeze and dyspnoea) on the day of BAL. All BALs were performed while the child was clinically stable and fit for general anaesthesia. The programme received approval from the Human Ethics Committee of Princess Margaret Hospital for Children (Perth, Australia) and parents/guardians gave written consent prior to each bronchoscopy.

Flexible bronchoscopy was performed via a laryngeal mask under general anaesthesia using i.v. propofol $\left(3-4 \mathrm{mg} \cdot \mathrm{kg}^{-1}\right)$, following pre-medication with midazolam $\left(0.5 \mathrm{mg} \cdot \mathrm{kg}^{-1}\right)$ if required. Suction through the bronchoscope was delayed until the tip passed the carina. With the bronchoscope wedged into the right middle lobe bronchus, three separate aliquots of normal saline $\left(1 \mathrm{~mL} \cdot \mathrm{kg}^{-1}\right)$ were instilled and retrieved. Additional BAL fluid (BALF) was obtained from the left lingular or the most abnormal lobe seen radiologically in most children. The first aspirate from each site was sent for microbiological analysis and the second and third aspirates from the right middle lobe were put on ice and processed immediately for assessment of inflammation.

\section{Microbiology}

BALF was cultured and $P$. aeruginosa identified using standard microbiological protocols [4]. A bacterial density of $>10^{4} \mathrm{cfu} \cdot \mathrm{mL}^{-1}$ was reported as infection and $10^{2}-$ $10^{4} \mathrm{cfu} \cdot \mathrm{mL}^{-1}$ as isolated colonies. BALF culturing mixed oral flora or $<10^{2} \mathrm{cfu} \cdot \mathrm{mL}^{-1}$ were classified as uninfected. $P$. aeruginosa colonies were described as mucoid, smooth or rough according to their appearance on horse blood agar. The routine sensitivity testing of these isolates included ciprofloxacin, ceftaxime, ticarcillin clavulanate, tobramycin and ceftazidime. Additional sensitivity testing was undertaken as required.

\section{BAL inflammatory markers}

BALF was processed immediately [4], with total and differential cell counts performed and supernatant frozen for subsequent cytokine analyses. Interleukin (IL)-8 was measured using ELISA (BD OptEIA; BD Biosciences, San Diego, CA, USA; working range $\left.0.01-6.40 \mathrm{ng} \cdot \mathrm{mL}^{-1}\right)$. A cytometric bead array (BD Cytometric Bead Array Human Inflammation kit; BD Biosciences) was used to measure IL-1 $\beta$ and IL-6 (ranges 20-5,000 pg. $\mathrm{mL}^{-1}$ ).

\section{Free neutrophil elastase}

Neutrophil elastase (NE) activity was measured using synthetic substrate N-methoxysuccinyl-ala-ala-pro-valp-nitroanilide (Sigma Aldrich, Steinheim, Germany) [23]. Human NE (Calbiochem, La Jolla, CA, USA) was used as the standard and limits of detection were $0.01-12 \mu \mathrm{g} \cdot \mathrm{mL}^{-1}$. This activity assay reflected the levels of free (unbound) NE and indicated that the anti-protease binding capacity was exceeded. Results are reported as a binary variable (detectable/nondetectable) and actual levels are given for those with detectable levels.

\section{P. aeruginosa eradication}

Children infected with $P$. aeruginosa underwent a standardised eradication protocol, including twice-daily chest physiotherapy, 14 days of i.v. tobramycin $\left(7.5 \mathrm{mg} \cdot \mathrm{kg}^{-1} \cdot \mathrm{day}^{-1}\right)$ and ticarcillin clavulanate $\left(300 \mathrm{mg} \cdot \mathrm{kg}^{-1} \cdot \mathrm{day}^{-1}\right)$ or ceftazidime $\left(150 \mathrm{mg} \cdot \mathrm{kg}^{-1} \cdot \mathrm{day}^{-1}\right)$, followed by 1 month of nebulised tobramycin ( $80 \mathrm{mg}$ in $2 \mathrm{~mL}$ b.i.d.) and oral ciprofloxacin $\left(10 \mathrm{mg} \cdot \mathrm{kg}^{-1}\right.$ b.i.d.). The absence of $P$. aeruginosa in BAL repeated 3 months after initial detection indicated successful eradication. Persistence of $P$. aeruginosa prompted a second eradication cycle. Re-infection with $P$. aeruginosa following a period of eradication was treated the same way as first acquisition.

\section{Statistics}

Repeat-measures ANOVA was used to determine the effect of $P$. aeruginosa acquisition and eradication on longitudinal measures of inflammation and in the comparison group of uninfected children. An unpaired t-test or Fisher's exact test were used to compare pulmonary inflammation between those infected with $P$. aeruginosa, those infected with organisms other than $P$. aeruginosa and uninfected children.

\section{RESULTS}

\section{Acquisition of $\mathbf{P}$. aeruginosa}

The present study includes data from the onset of the BAL programme in 1999 until March 2008. During this time 116 children completed or were engaged in the BAL programme and 408 BALs were performed for microbiological surveillance. The median (range) age of the children at the end of the observation period was $6.93(0.36-13.82)$ yrs, with the last BAL being performed at age $4.4(0.13-6.92)$ yrs and the number of BALs performed on individual children ranging from 1 to 9 (median 3.0). Overall, $27 \%$ of all surveillance BALs were positive for infection and $57 \%$ of children had at least one infection detected by BAL within the first 6 yrs of life. The common organisms isolated were Staphylococcus aureus (27 (23\%) out of 116); Haemophillus influenzae (12 (10\%) out of 116$)$, Stenotrophomonas maltophilia (nine (8\%) out of 116), Escherichia coli (six (5\%) out of 116), Streptococcus pneumoniae (five (4\%) out of 116) and Candida species (five (4\%) out of 116). P. aeruginosa was isolated from $33(28.4 \%)$ children (table 1$)$. The median (range) age at first detection of $P$. aeruginosa was 30.5 (3.371.4) months.

Since the introduction of NBS in 2000, 77 children diagnosed following detection by NBS took part in the BAL programme, and $21(27 \%)$ children were infected with $P$. aeruginosa. For three children aged 3.3, 3.8 and 7.9 months, $P$. aeruginosa was

\begin{tabular}{|c|c|c|}
\hline TABLE 1 & \multicolumn{2}{|c|}{$\begin{array}{l}\text { Characteristics of children who acquired } \\
\text { pulmonary infection with Pseudomonas } \\
\text { aeruginosa in the first } 6 \text { yrs of life }\end{array}$} \\
\hline \multicolumn{2}{|l|}{ Subjects $n$} & 33 \\
\hline \multicolumn{2}{|l|}{ Male } & $17(52)$ \\
\hline \multicolumn{2}{|c|}{ Genotype homozygote $\Delta F 508$} & $17(52)$ \\
\hline \multicolumn{2}{|c|}{ Diagnosis via new born screening } & $21(64)$ \\
\hline \multicolumn{2}{|c|}{ Age at first BAL months } & $3.1(0.7-58.2)$ \\
\hline \multicolumn{2}{|c|}{ Age at $P$. aeruginosa detection months } & $30.5(3.3-71.4)$ \\
\hline \multicolumn{2}{|c|}{ Mucoid $P$. aeruginosa at first detection } & $6(18.2)$ \\
\hline \multicolumn{2}{|c|}{ Age at mucoid $P$. aeruginosa detection months } & $40.0(15.7-70.5)$ \\
\hline \multicolumn{2}{|c|}{ Additional pathogens ${ }^{\#}$} & $16(48.5)$ \\
\hline \multicolumn{2}{|c|}{ Respiratory symptoms at $P$. aeruginosa detection } & $16(48.5)$ \\
\hline \multicolumn{3}{|c|}{$\begin{array}{l}\text { Data are presented as median (range) or } n(\%), \text { unless otherwise stated. BAL: } \\
\text { bronchoalveolar lavage. }{ }^{\#} \text { : presence of additional pathogens in BAL at time of } \\
\text { initial } P \text {. aeruginosa detection. }\end{array}$} \\
\hline
\end{tabular}


isolated on the first BAL and the median (range) age of first detection was 26.1 (3.3-65.2) months.

The density of $P$. aeruginosa was $>10^{4} \mathrm{cfu} \cdot \mathrm{mL}^{-1}$ in $84.8 \%$ of isolates (table 2). All isolates were sensitive on routine testing to a range of anti-pseudomonal antibiotics, including quinolones, $\beta$-lactams and aminoglycosides. $P$. aeruginosa was of mucoid phenotype at first detection in six children (age 40.0 (15.7-70.5) months).

P. aeruginosa was the sole organism in $16(48.5 \%)$ children, with $S$. aureus being the most common $(n=6)$ additional organism. Other co-cultured organisms included: Burkholderia cepacia $(\mathrm{n}=1)$; Aspergillus species $(\mathrm{n}=3) ; H$. influenzae $(\mathrm{n}=2) ; S$. maltophilia $(\mathrm{n}=2)$; Candida species $(\mathrm{n}=2)$; Alcaligenes $x$ ylosoxidans $(\mathrm{n}=1)$; S. pneumoniae $(\mathrm{n}=1)$; and mixed oral flora $(\mathrm{n}=3)$. Just under one half $(48.4 \%)$ of children had respiratory symptoms, mainly moist cough, at the time of isolation.

In total, $60 \%$ (245 out of 408 ) of the BALs were performed while children were on anti-Staphylococcal prophylaxis with amoxicillin clavulanate as per clinic protocol. There were no differences in rate of infection or infection with specific organisms between those children on prophylaxis and those not receiving antibiotics (table 3). Specifically, there was no difference in the numbers infected with $P$. aeruginosa (11.0 versus $8.0 \% ; \mathrm{p}=0.31$ ) nor in the median (range) age of acquisition of $P$. aeruginosa (30.9 (3.4-71.4) versus 35.5 (6.865.6) months; $\mathrm{p}=0.74$ ).

\section{Pulmonary inflammation}

Table 4 shows pulmonary inflammation in children at the time of detection of $P$. aeruginosa and in two comparison groups of similar ages selected from the clinic population. Children infected with $P$. aeruginosa had a higher inflammatory burden than children infected with organisms other than $P$. aeruginosa or than uninfected children (table 4). In addition, all children infected with $P$. aeruginosa had detectable levels of the proinflammatory cytokines IL-6 and IL- $1 \beta$ (mean \pm SD $1.75 \pm 0.52$ and $1.97 \pm 0.70 \log \left(\mathrm{pg} \cdot \mathrm{mL}^{-1}\right)$, respectively), whereas these two cytokines were only detectable in 34 and $31 \%$, respectively, of the uninfected group and in 55 and $52 \%$, respectively, of the group infected with organisms other than P. aeruginosa.

\section{P. aeruginosa eradication}

Five children had $P$. aeruginosa detected prior to the introduction of the eradication regimen in 2000 and three lacked posteradication BAL data. These children were excluded from the remaining analysis, leaving data from 26 children. All children received i.v. ticarcillin clavulanate and tobramycin (mean 14.8 days). Subsequent BAL data were available for a median (range) of 19 (3-69) months. P. aeruginosa was eradicated in 20 (77\%) out of 26 children following one eradication cycle. The six children who did not clear $P$. aeruginosa on the first cycle were older at detection (50.5 (40.8-62.5) months), three were diagnosed prior to the introduction of NBS and three cultured mucoid $P$. aeruginosa. Five underwent a second eradication cycle (i.v. ceftazidime and tobramycin based on physician preference and antibiotic sensitivities) with three successfully eradicating $P$. aeruginosa and one awaiting repeat BAL. The overall clearance rate was $88.5 \%$ ( 23 out of 26 ). One child failed to eradicate $P$. aeruginosa and the parents of another child declined a second eradication cycle. Both these children were diagnosed prior to NBS, enrolled onto the BAL programme at 40.3 and 58.2 months of age and isolated mucoid $P$. aeruginosa aged 58.4 and 70 months, respectively.

The median (range) duration of eradication was 19 (369) months (table 2). Four of the six children (aged 14, 14, 16

TABLE 2 Microbiological and clinical characteristics of pulmonary infection with Pseudomonas aeruginosa

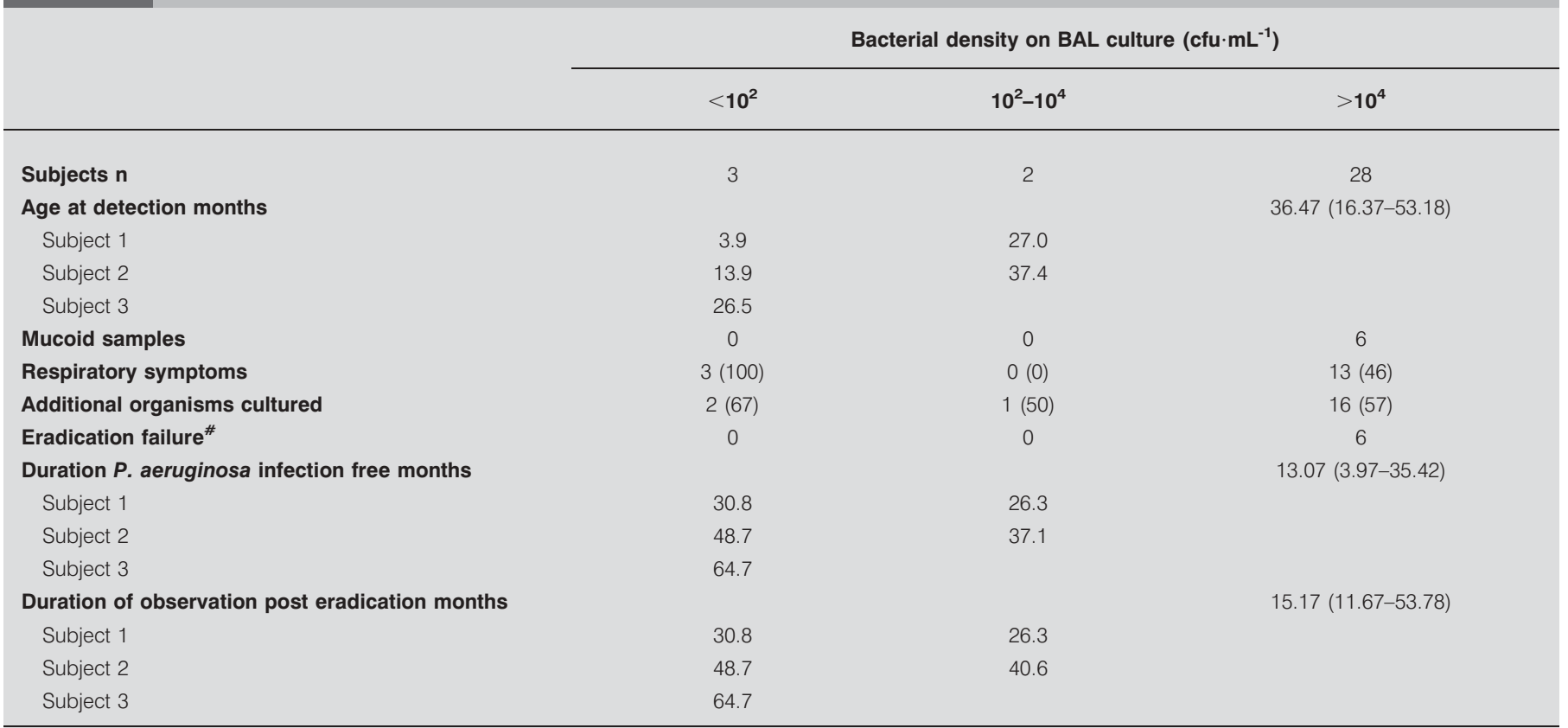

Data are presented as median (interquartile range) or $n(\%)$, unless otherwise stated. BAL: bronchoalveolar lavage. ${ }^{\#}$ : on the first cycle. 


\begin{tabular}{lcc}
\hline TABLE 3 & $\begin{array}{c}\text { Influence of anti-staphylococcal prophylaxis on } \\
\text { pulmonary infection }\end{array}$ \\
& Prophylaxis & No prophylaxis \\
\hline Subjects n & 245 & 163 \\
Age at BAL months & $2.3(0.07-6.85)$ & $2.8(0.06-6.92)$ \\
Any infection & $67(27.4)$ & $45(27.6)$ \\
Staphylococcus aureus & $15(6.1)$ & $17(10.4)$ \\
Pseudomonas aeruginosa & $27(11.0)$ & $13(8.0)$ \\
Haemophillus influenzae & $4(1.6)$ & $9(5.5)$ \\
Stenotrophomonas maltophilia & $4(1.63)$ & $4(2.5)$ \\
Escherichia coli & $6(2.5)$ & $1(0.6)$ \\
Streptococcus pneumoniae & $4(1.6)$ & $3(1.8)$ \\
Candida species & $6(2.5)$ & $1(0.6)$ \\
\hline
\end{tabular}

Data are presented as median (range) or $\mathrm{n}(\%)$, unless otherwise stated. BAL: bronchoalveolar lavage. Data are shown as number of BALs and no statistical differences were seen for any variable.

and 37 months) who isolated mucoid $P$. aeruginosa at first detection remained $P$. aeruginosa free for a median (range) of 42.5 (3-69) months. Three children had re-cultured $P$. aeruginosa, within 12, 33 and 37 months of eradication treatment.

There were no adverse events during bronchoscopy or related to antibiotic therapy. In addition, when specifically questioned 3-5 days after BAL, parents did not report fever $>38^{\circ} \mathrm{C}$ or prolonged cough post BAL. Tobramycin trough levels were within nontoxic ranges and renal function was normal in all children during treatment. Multiresistant $P$. aeruginosa was not isolated at any stage. Only two children acquired new organisms posteradication: S. maltophilia $(\mathrm{n}=1)$ and $S$. aureus $(\mathrm{n}=1)$.

Serial pulmonary inflammatory data prior, during and following $P$. aeruginosa eradication were available in 16 (10 males) out of 26 children with a median (range) age at first detection of 28.5 (19-38.5) months. All but one of these children remained $P$. aeruginosa infection free post eradication (median (range) follow-up BAL duration 14 (12-48.5) months). Complete inflammatory data (12 months prior to $P$. aeruginosa detection, at detection, 3 months following eradication, and 12 months post eradication) were available for 13 children (table 5). There were significant increases in neutrophils $(p=0.02)$, free NE $(p=0.001), \quad$ IL- $8 \quad(p=0.02)$ and IL-1 $\beta$ $(\mathrm{p}=0.01)$ in BALF at the time of $P$. aeruginosa detection (table 5) and a trend towards decreased levels of IL-1 $\beta(p=0.05)$ and free NE $(p=0.06) 3$ months later. Significant decreases in free $\mathrm{NE}(p=0.003)$ and IL-1 $\beta(p=0.003)$ were apparent 12 months post eradication, with similar trends in neutrophil number and IL-6. Levels of IL-8 continued to increase in the 3 months post eradication and were decreased to pre-infection levels at the 12-month post-eradication BAL (table 5). Table 5 also shows data from a comparison group of children of similar age selected from the clinic population who were uninfected on three successive annual BALs. These data show that there were no changes in pulmonary inflammation over the 3 yrs in the uninfected group. In addition, apart from a mild increase in neutrophils and IL-6, there were no differences in the pulmonary inflammation profile in the BAL performed 12 months prior to detection of $P$. aeruginosa compared with those not infected (table 5).

Serial inflammatory data were available in five out of six eradication first-treatment cycle failures. While numbers are too small for meaningful analysis, children who failed to eradicate $P$. aeruginosa had a trend towards a higher number of neutrophils when $P$. aeruginosa was detected compared with those in whom $P$. aeruginosa was successfully eradicated (354.7 versus $\left.149.8 \times 10^{3} \cdot \mathrm{mL}^{-1}\right)$ and higher neutrophil number (759 versus $\left.190.1 \times 10^{3} \cdot \mathrm{mL}^{-1}\right)$, more $\mathrm{NE}\left(1,580\right.$ versus $\left.200 \mathrm{ng} \cdot \mathrm{mL}^{-1}\right)$ and IL-1 $\beta$ ( 383.5 versus $\left.38.7 \mathrm{ng} \cdot \mathrm{mL}^{-1}\right)$ than those who cleared $P$. aeruginosa. The densities of $P$. aeruginosa in this group were $10^{5}$ $(\mathrm{n}=1), 10^{6}(\mathrm{n}=3)$ and $\geqslant 10^{7} \mathrm{cfu} \cdot \mathrm{mL}^{-1}(\mathrm{n}=1)$.

\section{DISCUSSION}

The data from the present study demonstrated that $28 \%$ of children with $\mathrm{CF}$ in Western Australia acquired pulmonary infection with $P$. aeruginosa in the first 6 yrs of life. The CF clinic in Perth is responsible for the care of all children with $\mathrm{CF}$

\begin{tabular}{|c|c|c|c|c|}
\hline ABLE 4 & & Uninfected & Infected, other than $P$. aeruginosa & Infected, $P$. aeruginosa \\
\hline \multicolumn{2}{|l|}{ Subjects $n$} & 33 & 33 & 33 \\
\hline \multicolumn{2}{|l|}{ Age yrs } & $2.55(1.13-4.09)$ & $2.51(1.33-4.07)$ & $2.54(1.31-4.09)$ \\
\hline \multicolumn{2}{|c|}{ Respiratory symptoms } & $5(15.2)$ & $8(24.2)$ & $16(48.5)^{\star \star}$ \\
\hline \multicolumn{2}{|c|}{ Neutrophils \% total cell count } & $14.2(6.8-26.5)$ & $32.5(9.5-55.9)$ & $37.5(23.9-68.4)^{\star \star}$ \\
\hline \multicolumn{2}{|c|}{$N E \log \left(\mu \mathrm{g} \cdot \mathrm{mL}^{-1}\right)$} & $-0.92 \pm 0.27$ & $-0.50 \pm 0.69$ & $0.22 \pm 0.94^{\star \star, ~ \# ~}$ \\
\hline \multicolumn{2}{|c|}{ NE present \% } & 9 & 41 & $75 * *, \#$ \\
\hline \multicolumn{2}{|c|}{ IL-8 $\log \left(\mathbf{n g} \cdot \mathrm{mL}^{-1}\right)$} & $-0.61 \pm 0.61$ & $-0.16 \pm 0.79$ & $0.15 \pm 0.71^{\star *}, \#$ \\
\hline
\end{tabular}

Data are presented as mean \pm SD or median (interquartile range), unless otherwise stated. NE: neutrophil elastase; IL: interleukin. ${ }^{* *}$ : $p<0.01 P$. aeruginosa versus uninfected group; ${ }^{*}: \mathrm{p}<0.01$ versus infected, other than $P$. aeruginosa. 
TABLE 5 Pulmonary inflammation at the time of Pseudomonas aeruginosa detection, 12 months prior to detection and 12 months post eradication

\begin{tabular}{|c|c|c|c|c|c|}
\hline \multicolumn{2}{|c|}{12 months prior } & \multicolumn{2}{|c|}{ P. aeruginosa detection } & \multicolumn{2}{|c|}{12 months post } \\
\hline $\begin{array}{l}\text { P. aeruginosa } \\
\text { group }^{\#}\end{array}$ & Not infected & $\begin{array}{l}\text { P. aeruginosa } \\
\text { group }\end{array}$ & Not infected & $\begin{array}{l}\text { P. aeruginosa } \\
\text { group }^{+}\end{array}$ & Not infected \\
\hline $1.41 \pm 1.27$ & $1.40 \pm 1.30$ & $2.45 \pm 1.31$ & $2.47 \pm 1.32$ & $3.50 \pm 1.27$ & $3.45 \pm 1.37$ \\
\hline 62 & 38 & 31 & 23 & 31 & 23 \\
\hline$-0.29 \pm 0.32$ & $-0.65 \pm 0.52$ & $-0.11 \pm 0.52$ & $-0.54 \pm 0.20$ & $-0.42 \pm 0.34$ & $-0.49 \pm 0.27$ \\
\hline $2.11 \pm 0.53^{*}$ & $1.60 \pm 0.45$ & $2.46 \pm 0.74 * * \star$ & $1.56 \pm 0.48$ & $2.00 \pm 0.55$ & $1.58 \pm 0.54$ \\
\hline $29.3 \pm 15.1$ & $22.0 \pm 16.3$ & $44.0 \pm 25.4^{*}$ & $16.8 \pm 12.4$ & $33.9 \pm 24.3$ & $17.9 \pm 16.8$ \\
\hline$-0.58 \pm 0.63$ & $-0.90 \pm 0.21$ & $0.21 \pm 0.91^{\star \star \star}$ & $-1 \pm 0$ & $-0.56 \pm 0.64$ & $-0.84 \pm 0.32$ \\
\hline 36 & 27 & $77^{\star \star \star}$ & 0 & 38 & 23 \\
\hline $0.03 \pm 0.52$ & $-0.88 \pm 0.51$ & $0.10 \pm 0.70^{*}$ & $-0.73 \pm 0.78$ & $0.12 \pm 0.65^{*}$ & $-0.59 \pm 0.73$ \\
\hline $1.49 \pm 0.38$ & $1.36 \pm 0.40$ & $1.90 \pm 0.55^{\star}$ & $1.30 \pm 0.30$ & $1.28 \pm 0.36$ & $1.20 \pm 0.34$ \\
\hline $1.67 \pm 0.35^{*}$ & $1.26 \pm 0.35$ & $1.78 \pm 0.50^{\star \star \star}$ & $1.13 \pm 0.33$ & $1.49 \pm 0.39$ & $1.29 \pm 0.38$ \\
\hline
\end{tabular}

Data are presented as mean \pm SD, unless otherwise stated. NE: neutrophil elastase; IL: interleukin. Data are from 13 children infected with $P$. aeruginosa and from 13 children of similar age who were not infected over this 3-yr period. ${ }^{*}$ : three children were infected with organisms other than $P$. aeruginosa: Haemophillus influenzae ( $n=1$ ), Streptococcus pneumoniae $(n=1)$ and Staphylococcus aureus $(n=1) ; "$ : two children also grew additional organisms: H. influenzae $(n=1)$ and $S$. aureus $(n=1) ;{ }^{+}:$two children were infected, one with $P$. aeruginosa (re-acquisition) and one with $S$. aureus. *: $p<0.05$ compared with uninfected group; *: $p<0.001$ compared with uninfected group.

in Western Australia, serving a population of approximately 2 million. The bronchoscopy-based pulmonary surveillance programme is directed towards the entire clinic population $<6$ yrs of age, with $>95 \%$ of the families participating. These data demonstrate that children with pulmonary infection with $P$. aeruginosa may be asymptomatic despite the presence of a significant microbiological burden. Bacterial densities of $P$. aeruginosa in all but five isolates were $>10^{4} \mathrm{cfu} \cdot \mathrm{mL}^{-1}$; indicative of lower respiratory infection [21]. In addition, the current authors have demonstrated that, in $77 \%$ of infected children, $P$. aeruginosa could be eradicated following a regimen of 2 weeks of i.v. followed by 4 weeks of nebulised and oral antipseudomonal antibiotics. Three additional children were cleared of infection following a second treatment cycle. The median (range) duration of eradication was 19 (3-69) months and was limited by the duration of follow-up available. These data demonstrate that early aggressive treatment can eradicate pulmonary infection with $P$. aeruginosa for a considerable time.

The relationship between pulmonary infection with $P$. aeruginosa and respiratory symptoms was poor in the present study, with $>50 \%$ being asymptomatic regardless of bacterial density (table 2). Given that BAL was performed annually, the present authors were not able to determine whether children were symptomatic when they first acquired P. aeruginosa (as opposed to when it was detected). A poor correlation between respiratory symptoms and the presence of pulmonary infection in the preschool population has previously been reported [4], highlighting the importance of routine surveillance in addition to respiratory cultures at pulmonary exacerbation.

Previous reports of $P$. aeruginosa acquisition have described an initial infection of low bacterial density with a nonmucoid phenotype $[10,24]$. In the present population, $84.8 \%$ of isolates were $>10^{4} \mathrm{cfu} \cdot \mathrm{mL}^{-1}$, with a relatively high prevalence $(18.2 \%)$ of mucoid $P$. aeruginosa. As the current data come from a population-based surveillance programme, it is interesting to speculate that the notion of initial infection with nonmucoid organisms transforming over time to mucoid phenotype under environmental pressure [25] may not be universally applicable in young children. Alternatively, a rapid phenotypic change from nonmucoid to mucoid within a 12-month period between BALs may have occurred in contrast to previous reports observing mucoid transition to be a slow process in children [10]. No differences were found in the age of detection and in bacterial densities between the nonmucoid and mucoid phenotypes, supporting the concept of primary acquisition of mucoid $P$. aeruginosa in some children. While the present authors do not have definitive data proving primary acquisition of mucoid $P$. aeruginosa, these data highlight the need for more frequent pathogen surveillance using techniques that accurately reflect pulmonary infection.

The data from the present study suggest that eradication of $P$. aeruginosa is attainable in young children using a combination of i.v. and oral/nebulised antibiotic therapy. The success rate observed in the current study following one eradication cycle is similar to that reported by GIBSON et al. [26] using nebulised tobramycin, although the present authors had slightly greater success in eradicating mucoid $P$. aeruginosa. The current data also suggest that a more aggressive approach with a second eradication cycle may be necessary for some children to clear $P$. aeruginosa. Re-infection was less frequent in the present study than in previous reports of combined i.v. and oral/ nebulised therapy $[11,15]$ and the potential for children to remain free of $P$. aeruginosa for as long as 69 months was observed, limited primarily by the duration of follow-up available. 
Only two studies to date have examined the use of $i . v$. antibiotics combined with nebulised and oral antibiotics and report only transient eradication [11, 15]. NIXON et al. [11] observed $P$. aeruginosa eradication for longer than 12 months in only six ( $25 \%$ ) out of 24 children aged $<7$ yrs with $P$. aeruginosa detected by BAL $(n=17)$ or sputum $(n=6)$. Lower eradication rates in that study may reflect, in part, a higher proportion (54\%) of children with mucoid $P$. aeruginosa at first detection, and the subsequent recognition of a virulent epidemic strain of $P$. aeruginosa among the cohort. MuNCK et al. [15] observed eradication of $P$. aeruginosa (based on negative oropharyngeal (OP) cultures) for a mean duration of 8.6 months in 14 children $<6$ yrs of age, following combination i.v. and nebulised antibiotic therapy. All children re-colonised within 2 yrs. OP cultures are not routinely performed in the present authors' clinic, thus limiting the ability to compare current data with those from other clinics.

The present study was too small to determine factors that predicted eradication failure or success, although those who failed to clear on the first treatment cycle were somewhat older, with greater bacterial densities and higher BAL inflammatory markers at the time of $P$. aeruginosa detection. Three of the six children who cultured mucoid $P$. aeruginosa did not clear with the first cycle, although one child cleared with a second cycle. The present authors did not prospectively evaluate adherence to oral and nebulised antibiotics and, therefore, cannot assess the contribution of adherence to eradication success. A larger, multicentre study, preferably performed in children diagnosed following detection by NBS, will be required to adequately determine factors that predict eradication failure.

The numbers of children with serial pulmonary inflammatory data in the present study were small and results should be interpreted cautiously. Within these limitations, current findings suggest that clearance of $P$. aeruginosa from the lower respiratory tract following combination i.v. and oral/nebulised antibiotic therapy was associated with a reduction in pro-inflammatory IL$1 \beta$, neutrophil numbers and activity by 3 months which persisted to 12 months post eradication (table 5). Interestingly, the stimulus for neutrophil migration into the lung, IL-8, persisted at 3 months post-eradication and was not observed to return towards pre-infection levels until 12 months post eradication treatment. Only one other published study has described the effect of $P$. aeruginosa eradication on pulmonary inflammation [26]. In that report inflammatory markers were only studied at the time of $P$. aeruginosa detection and up to 16 weeks post eradication therapy and while reduced neutrophil levels were observed in some subjects, no consistent trends in other inflammatory markers were apparent.

There are a number of potential limitations of the present BAL programme that need to be acknowledged. As bronchoscopy is an invasive procedure requiring general anaesthesia, the frequency of BAL is restricted, which may delay detection of the $P$. aeruginosa infection and may mean that transient infection/re-infection could be missed. Thus, the prevalence of pulmonary infection with $P$. aeruginosa may be underestimated in the present study population. Delay in detecting $P$. aeruginosa and delay in treatment initiation may explain the observations of higher bacterial densities and prevalence of mucoid phenotype in the current study. It was recognised that a negative BAL culture, or the absence of a particular organism on culture, does not give an unequivocal assurance that the organism is not present in the lungs [27]. In addition, as essentially the present entire clinic population under the age of $6 \mathrm{yrs}(>95 \%$ of eligible families) participated in the BAL-based surveillance programme, no control group was available to demonstrate that there were no adverse effects associated with the programme. The best option available is to compare current data with data available from the Australian National CF Data Registry [20]. These data are not directly comparable, as the age groups reported are $0-4$ and 5$9 \mathrm{yrs}$, however they show infection rates with $P$. aeruginosa of 16.3 and $35.8 \%$, respectively [20]. Clinic-specific data show that children aged 6-10 yrs managed at Princess Margaret Hospital are ranked among the highest for weight (fourth overall with $25 \%$ having weight $<25$ th percentile), height (second overall, with $30 \%$ having height $<25$ th percentile) and lung function (ranked first overall for forced expiratory volume in one second). These data suggest that children who have participated in the current BAL programme do at least as well as children managed in other Australian clinics.

In conclusion, data presented from the current BAL-based surveillance programme demonstrate that $28 \%$ of children $<6$ yrs old acquired pulmonary infection with $P$. aeruginosa. Children as young as 3 months old detected by NBS may be infected with $P$. aeruginosa. Infection with mucoid $P$. aeruginosa and higher bacterial densities were more common than previously appreciated and detected in the absence of respiratory symptoms. Clearance of moderate-to-high bacterial loads of $P$. aeruginosa together with a reduction in pulmonary inflammation, is achievable for considerable periods in young children and infants using a combination of i.v. and oral/ nebulised antibiotic therapy.

The present study suggests that a proportion of children may require and respond to more aggressive eradication therapy. A larger study is needed to further characterise factors that predict response to Pseudomonas aeruginosa eradication therapy so that individualised and potentially more aggressive treatment may be directed to those considered at greater risk of eradication failure.

\section{ACKNOWLEDGEMENTS}

The present authors would like to thank the following for their contribution to the cystic fibrosis bronchoalveolar programme during the past 7 yrs: A. Franzmann, S. Turner, A. Moller, F. Horak, A. Martin, A. Schultz and D. Mullane (Dept of Respiratory Medicine, Princess Margaret Hospital for Children, Perth, Australia); C. Calogero and P. Pitrez (Division of Clinical Sciences, Telethon Institute for Child Health Research, Perth). The current authors would also like to acknowledge the invaluable services of E. Balding (Dept of Respiratory Medicine, Princess Margaret Hospital for Children), K. Winfield and S. Gard (Division of Clinical Sciences, Telethon Institute for Child Health Research).

\section{REFERENCES}

1 Accurso FJ. Early pulmonary disease in cystic fibrosis. Curr Opin Pulm Med 1997; 3: 400-403.

2 Armstrong DS, Grimwood K, Carlin JB, et al. Lower airway inflammation in infants and young children with cystic fibrosis. Am J Respir Crit Care Med 1997; 156: 1197-1204. 
3 Khan TZ, Wagener JS, Bost T, Martinez J, Accurso FJ, Riches DW. Early pulmonary inflammation in infants with cystic fibrosis. Am J Respir Crit Care Med 1995; 151: 10751082.

4 Brennan S, Hall GL, Horak F, et al. Correlation of forced oscillation technique in preschool children with cystic fibrosis with pulmonary inflammation. Thorax 2005; 60: 159-163.

5 Wagener JS, Kahn TZ, Copenhaver SC, Accurso FJ. Early inflammation and the development of pulmonary disease in cystic fibrosis. Pediatr Pulmonol Suppl 1997; 16: 267-268.

6 Emerson J, Rosenfeld M, McNamara S, Ramsey B, Gibson RL. Pseudomonas aeruginosa and other predictors of mortality and morbidity in young children with cystic fibrosis. Pediatr Pulmonol 2002; 34: 91-100.

7 Kosorok MR, Zeng L, West SE, et al. Acceleration of lung disease in children with cystic fibrosis after Pseudomonas aeruginosa acquisition. Pediatr Pulmonol 2001; 32: 277-287.

8 Cystic Fibrosis Foundation. Patient Registry Annual Data Report 2006. Bethesda, 2008. Available from www.cff.org/ UploadedFiles/research/ClinicalResearch/2006\%20Patient \%20Registry\%20Report.pdf Date last accessed: September 23, 2008.

9 Hudson VL, Wielinski CL, Regelmann WE. Prognostic implications of initial oropharyngeal bacterial flora in patients with cystic fibrosis diagnosed before the age of two years. J Pediatr 1993; 122: 854-860.

$10 \mathrm{Li} \mathrm{Z,} \mathrm{Kosorok} \mathrm{MR,} \mathrm{Farrell} \mathrm{PM,} \mathrm{et} \mathrm{al.} \mathrm{Longitudinal}$ development of mucoid Pseudomonas aeruginosa infection and lung disease progression in children with cystic fibrosis. JAMA 2005; 293: 581-588.

11 Nixon GM, Armstrong DS, Carzino R, et al. Clinical outcome after early Pseudomonas aeruginosa infection in cystic fibrosis. J Pediatr 2001; 138: 699-704.

12 Gibson RL, Emerson J, McNamara S, et al. Significant microbiological effect of inhaled tobramycin in young children with cystic fibrosis. Am J Respir Crit Care Med 2003; 167: 841-849.

13 Griese M, Muller I, Reinhardt D. Eradication of initial Pseudomonas aeruginosa colonization in patients with cystic fibrosis. Eur J Med Res 2002; 21: 79-80.

14 Lee TW, Brownlee KG, Denton M, Littlewood JM, Conway SP. Reduction in prevalence of chronic Pseudomonas aeruginosa infection at a regional pediatric cystic fibrosis center. Pediatr Pulmonol 2004; 2: 104-110.

15 Munck A, Bonacorsi S, Mariani-Kurkdjian P, et al. Genotypic characterization of Pseudomonas aeruginosa strains recovered from patients with cystic fibrosis after initial and subsequent colonization. Pediatr Pulmonol 2001; 32: 288-292.
16 Taccetti G, Campana S, Festini F, Mascherini M, Doring G. Early eradication therapy against Pseudomonas aeruginosa in cystic fibrosis patients. Eur Respir J 2005; 26: 458-461.

17 Frederiksen B, Koch C, Hoiby N. Antibiotic treatment of initial colonization with Pseudomonas aeruginosa postpones chronic infection and prevents deterioration of pulmonary function in cystic fibrosis. Pediatr Pulmonol 1997; 23: 330-335.

18 The French Cystic Fibrosis Registry. Report on 2005 data of the National Cystic Fibrosis Observatory. Paris 2007. Available from www.vaincrelamuco.org/e_upload/pdf/ rfm_report_on_2005_data_in_english.pdf Date last accessed: September 23, 2008.

19 UK Cystic Fibrosis Trust. CF Registry - Annual Data Reports. Available from www.cftrust.org.uk/aboutcf/ publications/cfregistryreports / Date last accessed: September 23, 2008.

20 Cystic Fibrosis in Australia. Australian National Cystic Fibrosis Data Registry - 2005. Available from www. cysticfibrosis.org.au/projects/dataregistry/ Date last accessed: September 23, 2008.

21 Armstrong DS, Grimwood K, Carlin JB, Carzino R, Olinsky A, Phelan PD. Bronchoalveolar lavage or oropharyngeal cultures to identify lower respiratory pathogens in infants with cystic fibrosis. Pediatr Pulmonol 1996; 21: 267-275.

22 Rosenfeld M, Emerson J, Accurso F, et al. Diagnostic accuracy of oropharyngeal cultures in infants and young children with cystic fibrosis. Pediatr Pulmonol 1999; 28: 321-328.

23 Delacourt C, Herigault S, Delclaux C, et al. Protection against acute lung injury by intravenous or intratracheal pretreatment with EPI-HNE-4, a new potent neutrophil elastase inhibitor. Am J Respir Cell Mol Biol 2002; 26: 290-297.

24 Burns JL, Gibson RL, McNamara S, et al. Longitudinal assessment of Pseudomonas aeruginosa in young children with cystic fibrosis. J Infect Dis 2001; 183: 444-452.

25 Speert DP, Farmer SW, Campbell ME, Musser JM, Selander RK, Kuo S. Conversion of Pseudomonas aeruginosa to the phenotype characteristic of strains from patients with cystic fibrosis. J Clin Microbiol 1990; 2: 188-194.

26 Gibson RL, Emerson J, Mayer-Hamblett N, et al. Duration of treatment effect after tobramycin solution for inhalation in young children with cystic fibrosis. Pediatr Pulmonol 2007; 42: 610-623.

27 Gutierrez JP, Grimwood K, Armstrong DS, et al. Interlobar differences in bronchoalveolar lavage fluid from children with cystic fibrosis. Eur Respir J 2001; 17: 281-286. 